\title{
Pediatric Choanal Atresia: Our Experience
}

\author{
${ }^{1}$ Mohnish Grover, ${ }^{2}$ Gaurav Gupta, ${ }^{3}$ Shruti Bhargava, ${ }^{4}$ Shashank Nath Singh, ${ }^{5}$ Mahendra S Hada \\ ${ }^{6}$ Beni Prasad, ${ }^{7}$ Man Prakash Sharma
}

\begin{abstract}
Objective: To discuss our experience with choanal atresia and factors affecting the results.

Materials and methods: A multicentric prospective study of patients diagnosed with choanal atresia from April 2008 to J anuary 2014 at SMS Medical College, J aipur and SP Medical College, Bikaner was conducted. All patients were evaluated by CT PNS and then operated by transnasal endoscopic route. Stents were placed in all patients. Patients were followed up for an average of 3.5 years (range 9 months to 5.5 years).
\end{abstract}

Results: Nineteen cases were included: 24 sides were operated. No intraoperative complications were there. A restenosis rate of $29.1 \%$ (7 out of 24 ) was found in our series requiring revision surgery.

Conclusion: Endoscopic transnasal approach is a safe and efficacious option for treatment of choanal atresia. Use of stent in our series was not associated with high rate of restenosis.

Keywords: Choanal, Atresia, Management, Endoscopic.

How to cite this article: Grover M, Gupta G, Bhargava S, Singh SN, Hada MS, Prasad B, Sharma MP. Pediatric Choanal Atresia: O ur Experience. Clin R hinol An Int J 2014;7(1):13-15.

\section{Source of support: Nil}

\section{Conflict of interest: None}

\section{INTRODUCTION}

Posterior choanal atresia, or commonly called as choanal atresia, is an entity recognized for over 200 years. In 1755 , Roederer first described congenital choanal atresia. In 1854 , Emmert reported the first successful surgical procedure for congenital choanal atresia in a 7-year-old boy using a curved trocar transnasally. However, till date the results of treatment of this condition have been variable.

The average rate of choanal atresia is 0.82 cases per 10,000 individuals. Unilateral atresia occurs more frequently

\footnotetext{
${ }^{1-5}$ Assistant Professor, ${ }^{6,7}$ P rofessor

${ }^{1,4-7}$ Department of ENT, SMS Medical College, J aipur, R ajasthan India

${ }^{2}$ Department of ENT, SP Medical College, Bikaner, Rajasthan India

${ }^{3}$ Department of Pathology, SMS Medical College, J aipur Rajasthan, India
}

Corresponding Author: Mohnish Grover, Assistant P rofessor Department of ENT, 8/250, Malviya Nagar, J aipur, Rajasthan India, Phone: 09461306200, e-mail: drmohnish_aiims@ rediffmail.com on the right side. The ratio of unilateral to bilateral cases is $2: 1$.

In this study, we discuss the demographic profile, management and results of cases presenting to out institute.

\section{MATERIALS AND METHODS}

A prospective study was conducted in Department of Otolaryngology and Head and Neck Surgery, SMS Medical College, Jaipur and SP Medical College, Bikaner from April 2008 to January 2014. Patients presenting directly to our department or those referred from pediatrics were included in study. The clinical evaluation included a complete physical examination to assess for other congenital anomalies. In young patients a small feeding tube was used to determine the patency of the choana, but a complete nasal and nasopharyngeal examination was performed using a flexible fiberoptic endoscope to assess the deformity. They were evaluated by nasal endoscopy and noncontrast computed tomography of nose and paranasal sinuses. Depending on the unilaterality, age of patient and type of choanal atresia, patients were planned for surgery. All patients were operated by transnasal endoscopic approach. In cases with mixed choanal atresia, the membranous part was punctured by suction tip and then bony part was removed using curettes and microear cup forceps in young children and microdebrider, Blakesley forceps and Kerrisons punch in older children. In pure bony cases, posterior septectomy was done and bony part was removed along the most inferomedial aspect and then widened as appropriate. Packing was done with merocele which was removed after 72 hours. Stenting was done in all cases using age appropriate feeding tube. Nasal packing was removed after 72 hours and stents after 4 weeks. All patients were kept on antireflux management for 6 weeks to minimize granulation formation. Patients were regularly followed up with nasal endoscopy in outdoor setting. Patients were followed up for an average of 3.5 years (range 9 months to 5.5 years).

\section{RESULTS}

Nineteen cases with choanal atresia were included in this study; 11 being females and 8 males. Bilateral choanal atresia was found in 5 patients (Fig. 1) and 14 had unilateral pathology (Fig. 2). Thirteen patients had mixed bony membranous choanal atresia (see Fig. 2) 
while 6 had pure bony choanal atresia (see Fig. 1). All patients with bilateral choanal atresia were operated within 10 days of birth. Unilateral cases had an age range of 5 to 12 years with an average of 9.6 years.

All patients underwent surgery by transnasal endoscopic approach and stent was put (Fig. 3). There were no intraoperative complications. Out of 24 sides operated, 7 required revision surgery in form of removal of scar tissue or additional drilling of persistent bony stenosis.

\section{DISCUSSION}

Choanal atresia has been known to mankind even before we knew about many simple diseases affecting human body. However, due to rarity and complex nature of this condition, the treatment is still not very well-defined. The advent of nasal endoscopes has made the treatment more efficacious and safe. However, still use of stents, mitomycinc, LASER continue to be debated. The nasal cavities extend posteriorly during development under the influence of the posteriorly directed fusion of the palatal processes. Thinning of the membrane occurs, which separates the nasal cavities from the oral cavity. By the 38th day of development, the 2-layer membrane consisting of nasal and oral epithelia ruptures and forms the choanae (posterior nares). Failure of the bucconasal membrane of Hochstetter to rupture results in choanal atresia. Various etiological factors have been hypothesized. Barbero et $\mathrm{al}^{1}$ (2008), Kannan et $\mathrm{al}^{2}$ (2008) implicated prenatal use of antithyroid (methimazole, carbimazole) medications for causing choanal atresia.

Kancherla et $\mathrm{al}^{3}$ (2014) found evidence linking choanal atresia to maternal exposure to various nutrients, thyroid medications and cigarettes. Positive associations were found between the following prepregnancy exposures and choanal atresia: Intake in the highest quartile: Vitamin B12, zinc, niacin; Intake in the lowest quartile: Methionine, vitamin D; Coffee ( $\geq 3$ cups per day). Positive associations with thyroid medications and cigarette smoking were also found between periconceptual exposures and choanal atresia. Three of our cases had a history or prenatal antithyroid drug intake; 2 patients also had congenital heart disease.

Symptoms of severe airway obstruction and cyclical cyanosis are the classic signs of neonatal bilateral atresia. When crying alleviates respiratory distress in an obligate nasal breather, the neonatologist should be alerted to the probability of bilateral choanal atresia. This was present in all our bilateral cases. Unilateral atresia may not be detected for years, and patients may present with unilateral rhinorrhea or congestion. In our series, the most common presenting complaint was unilateral nasal obstruction. Treatment protocol of choanal atresia is as important as its diagnosis.

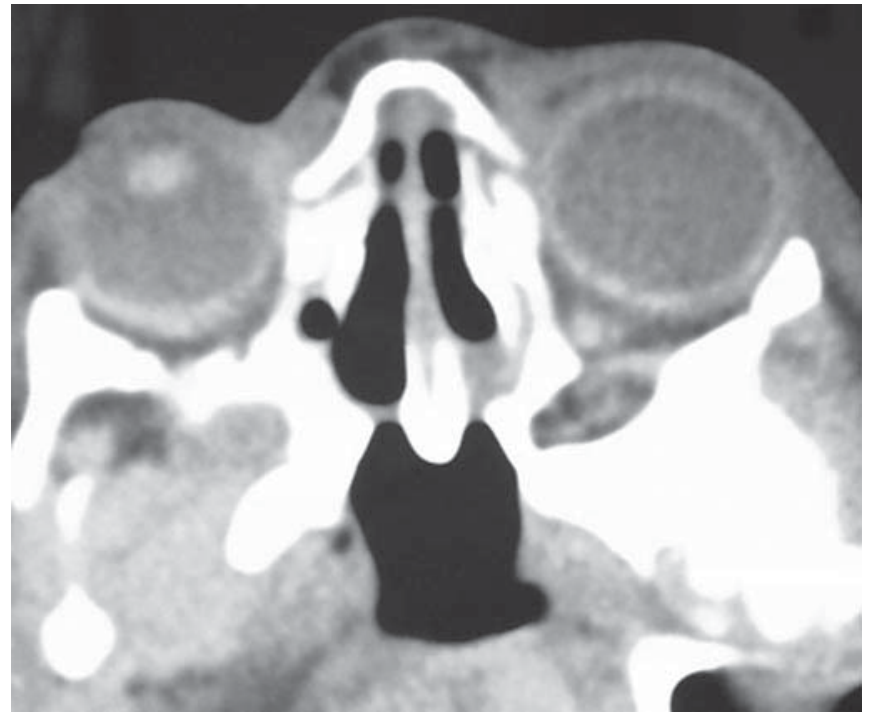

Fig. 1: CT PNS axial cut showing bilateral mixed type choanal atresia

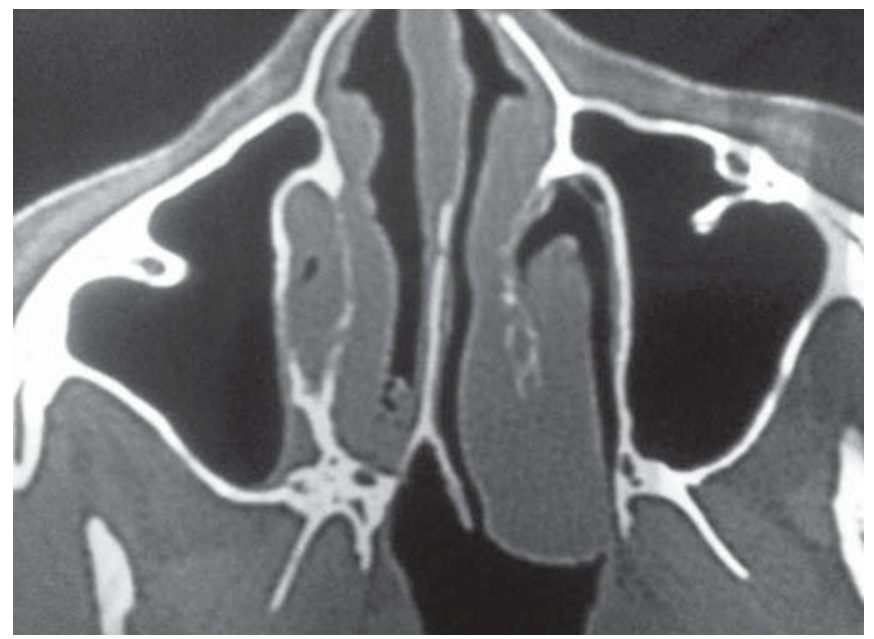

Fig. 2: CT PNS axial cut showing right bony choanal atresia

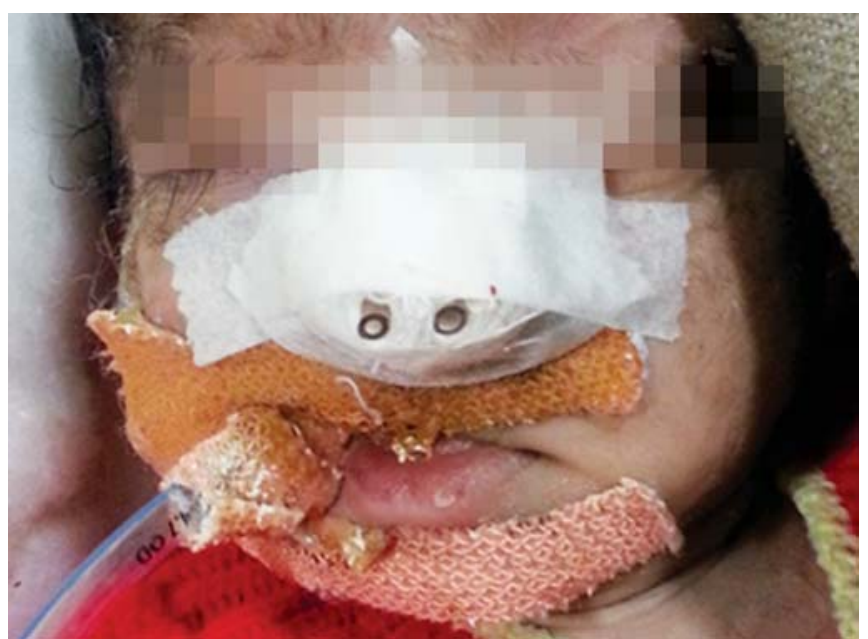

Fig. 3: Immediate postoperative photograph of neonate with nasal stents in both nasal cavities

Bilateral choanal atresia needs an emergency treatment for airway maintenance. Oral airway between the tongue and palate can be used for this purpose till definitive management 
is carried out. Definitive management includes transpalatal, trans-septal and transnasal approaches. Transnansal approach started with blind/ microscopic procedures. However, high recurrence rates made way for transpalatal approach. With use of endoscopes, endoscopic transnasal approaches have become treatment of choice. Technology advancements, like microdebrider and LASER, have made it even more effective. The endoscopic technique (nasal or retropalatal), with or without powered instrumentation, offers excellent visualization with great ease in removing the bony choanae. ${ }^{4}$

Carter et $\mathrm{al}^{5}$ recently published a landmark paper of a 20 years experience on choanal atresia. They reported 37 surgeries on 17 patients. They found significantly better results with mitomycin $\mathrm{C}$ application, with fewer revision surgeries (20\% vs none) and lesser incidence of granulation formation ( $53 \%$ vs $9 \%)$. They also found that greater amount of sides in the stent group developed granulation tissue $(50 \%$ vs $0 \% ; \mathrm{p}=0.023)$. The average number of procedures performed was significantly greater in those patients treated with a stent (2.33 vs 1.12; $p=0.008)$. The average time spent in the hospital was significantly shorter (7.09 vs 2.33 days; $\mathrm{p}=0.02)$ in those patients treated without stenting. However, they recommended that consideration should be given toward stent placement in all neonates for the prevention of postoperative airway obstruction. Our incidence of restenosis with stents has not been as high as reported in this article.

Another study by Newman et al, ${ }^{6}$ deals with 33 patients (31 endoscopic, 2 transnasal). Forty-three sides were operates endoscopically, 9 required revision surgeries. No significant difference was observed in the rate of restenosis among cases treated endoscopically with mitomycin C (22 of 43 operative sides, $p=0.13$ ), with stenting ( 36 of 43 operative sides, $\mathrm{p}=0.99)$, or with subsequent dilation $(\mathrm{p}=0.45)$.

De Freitas $\mathrm{RP}^{7}$ reported their results of transnasal endoscopic repair of bilateral choanal atresia. All patients were stented using modified endotracheal tubes for a mean of 75 days (range, 5-158 days). Of the 20 with adequate follow-up, 14 patients had successful primary repair (70\%).
Of the six requiring revision surgery, three had one revision procedure, and three had two revision procedures. All patients were free of recurrence at last follow-up. Mean follow-up after removal of primary stent was 25.2 months (range, 6.6-77.9 months). They concluded that stenting for 3 months was found to reduce the chance of early restenosis.

We have not used mitomycin $\mathrm{C}$ in our study and have used stents in all our cases and have had retenosis in 7 out of 24 sides $(29.1 \%)$.

\section{CONCLUSION}

Choanal atresia is an uncommon condition. With advent of endoscopes the results have dramatically changed. We recommend that all cases be operated by transnasal endoscopic approach and we have not found use of stents to give rise to high restenosis rates. We strongly recommend their use, specially in neonates.

\section{REFERENCES}

1. Barbero P, Valdez R, Rodríguez H, et al. Choanal atresia associated with maternal hyperthyroidism treated with methimazole: a casecontrol study. Am J Med Genet A 2008 Sep 15;146A(18):23902395.

2. Kannan L, Mishra S, Agarwal R, Kartikeyan V, Gupta N, Kabra M. Carbimazole embryopathy-bilateral choanal atresia and patent vitello-intestinal duct: a case report and review of literature. Birth Defects Res A Clin Mol Teratol 2008 Sep;82(9):649-651.

3. Kancherla V, Romitti PA, Sun L, et al. Descriptive and risk factor analysis for choanal atresia: The National Birth Defects Prevention Study, 1997-2007. Eur J Med Genet 2014 Feb 24.

4. Assanasen P, Metheetrairut C. Choanal atresia. J Med Assoc Thai 2009 May;92(5):699-706.

5. Carter JM, Lawlor C, Guarisco JL. The efficacy of mitomycin and stenting in choanal atresia repair: a 20 year experience. Int J Pediatr Otorhinolaryngol 2014 Feb;78(2):307-311.

6. Newman JR, Harmon P, Shirley WP, Hill JS, Woolley AL, Wiatrak BJ. Operative management of choanal atresia: a 15-year experience. JAMA Otolaryngol Head Neck Surg 2013 Jan;139(1):71-75.

7. De Freitas RP, Berkowitz RG. Bilateral choanal atresia repair in neonates: a single surgeon experience. Int J Pediatr Otorhinolaryngol 2012 Jun;76(6):873-878. 